\title{
Pacific
}

Journal of

Mathematics

\section{LARGE EIGENVALUES AND CONCENTRATION}

\author{
Bruno Colbois AND Alessandro Savo
}




\title{
LARGE EIGENVALUES AND CONCENTRATION
}

\author{
Bruno Colbois And Alessandro Savo
}

\begin{abstract}
Let $M^{n}=(M, g)$ be a compact, connected, Riemannian manifold of dimension $n$. Let $\mu$ be the measure $\mu=\sigma \operatorname{dvol}_{g}$, where $\sigma \in C^{\infty}(M)$ is a nonnegative density. We first show that, under some mild metric conditions that do not involve the curvature, the presence of a large eigenvalue (or more precisely of a large gap in the spectrum) for the Laplacian associated to the density $\sigma$ on $M$ implies a strong concentration phenomenon for the measure $\mu$. When the density is positive, we show that our result is optimal. Then we investigate the case of a Laplace-type operator $D=\nabla^{*} \nabla+T$ on a vector bundle $E$ over $M$, and show that the presence of a large gap between the $(k+1)$-st eigenvalue $\lambda_{k+1}$ and the $k$-th eigenvalue $\lambda_{k}$ implies a concentration phenomenon for the eigensections associated to the eigenvalues $\lambda_{1}, \ldots, \lambda_{k}$ of the operator $D$.
\end{abstract}

\section{Introduction}

The goal of this paper is to show that, under some mild metric conditions, the presence of a large eigenvalue of the Laplacian $\Delta$ on a compact Riemannian manifold $M$ implies that the Riemannian volume concentrates around a finite set of points. Actually, we show that a similar phenomenon holds for any Laplace-type operator $D$ acting on sections of a vector bundle on $M$, if one replaces the Riemannian volume by the squared norm of a first eigensection of $D$.

Let us recall briefly the main known facts about concentration and the spectrum of the Laplace operator. In what follows, we number the eigenvalues of $\Delta$ so that $\lambda_{1}(M)=0$ and $\lambda_{2}(M)$ is the first positive eigenvalue.

For a closed Riemannian manifold of dimension $n$ whose Ricci curvature is bounded below, that is, Ric $\geq-(n-1) a^{2}$, we have the following well-known inequality due to Cheng [1975]:

$$
\lambda_{k+1}(M) \leq \frac{(n-1)^{2} a^{2}}{4}+\frac{c(n) k^{2}}{\operatorname{diam}(M)^{2}},
$$

MSC2000: primary 58J50; secondary 35P15.

Keywords: eigenvalues, upper bounds, Laplace-type operators, concentration. 
where $c(n)$ is a constant depending only on $n$. This shows that when the $k$-th eigenvalue is very large, the whole manifold is contained in a small neighborhood of any of its points and so we have a strong concentration phenomenon.

At the other extreme, if we make no assumption other than compactness we still have a concentration phenomenon, first observed by Gromov and Milman [1983, Theorem 4.1]. It says that if $A$ is a closed subset with positive normalized measure $\mu(A)=\alpha$ and $r>0$, then

$$
\mu\left(A^{r}\right) \geq 1-\left(1-\alpha^{2}\right) \exp \left(-r \sqrt{\lambda_{2}(M)} \ln (1+\alpha)\right),
$$

where $A^{r}=\{x \in M: d(A, x)<r\}$.

So, when the first (positive) eigenvalue is large, almost all relative volume of $M$ lies in a small neighborhood of any set of fixed positive measure.

However, we stress that $\mu(A)$ being positive is essential in the estimate; the sole assumption that $\lambda_{2}(M)$ is large does not guarantee that the volume concentrates around, say, a finite set of points. For example, take $M_{n}$ to be the $n$-dimensional unit sphere. Then $\lambda_{2}\left(M_{n}\right)$ (which is equal to $n$ ) tends to infinity with $n$; we have concentration in the sense of Gromov and Milman, and yet the volume of $M_{n}$ is uniformly distributed and cannot concentrate around any finite set. In Section A.4 we will give another counterexample in which the dimension is fixed.

Inequality (1) can be generalized to the other eigenvalues using an interesting upper bound of $\lambda_{k}(M)$ due to Chung, Grigor'yan and Yau; the upper bound is given in terms of the least distance between $k$ mutually disjoint subsets of fixed positive measure; see [Chung et al. 1997] and also [Friedman and Tillich 2000] for a sharp estimate.

This paper deals with concentration around a finite number of points, and with a simple metric condition that will imply this phenomenon. Namely, we require that the number of balls of radius $r$ needed to cover a ball of radius $4 r$ is uniformly bounded above by a constant $C$ for $r \leq 1$. We then prove the following fact:

If the $(k+1)$-st eigenvalue of the Laplacian of $M$ is large, then most of the volume of $M$ concentrates near (at most) $k$ points of the manifold.

However, we will prove a result (Theorem 4) that is much more general; in particular, it will imply the following fact. Consider a Laplace-type operator $D$ acting on the sections of a smooth vector bundle on $M$ (for example, the Laplacian on forms, the square of the Dirac operator or the Schroedinger operator). Then:

If the gap between the $(k+1)$-st and the $k$-th eigenvalue of $D$ is large, then any eigensection associated to the first $k$ eigenvalues concentrates its $L^{2}$-norm near (at most) $k$ points of the manifold.

Both the above estimates depend explicitly on the constant $C$.

In the rest of the introduction we state the precise results: Theorems 1, 2 and 3. 
1.1. Some definitions. We will consider metric measure spaces $(M, \mu, d)$ of the following type:

- $M=\left(M^{n}, g\right)$ is a compact, connected Riemannian manifold of dimension $n$, possibly with nonempty boundary.

- $\mu$ is the measure $\mu=\sigma \mathrm{dvol}_{g}$, where $\sigma \in C^{\infty}(M)$ is a nonnegative density. We will also assume, without loss of generality, that $\mu$ is a probability measure, that is, $\int_{M} \sigma \mathrm{dvol}_{g}=1$.

- $d$ is a distance function that is assumed to be Lipschitz, that is, $|\nabla d| \leq 1$ almost everywhere with respect to $\mu$.

For $r>0$, define $C_{d}(M, r)$ to be the minimal number of balls of radius $r$ in $(M, d)$ needed to cover a ball of radius $4 r$. Then $C_{d}(M, r)$ is finite for all $r$.

We will set

$$
C_{d}(M)=\sup _{r \in(0,1]} C_{d}(M, r),
$$

and call it the packing constant of the pair $(M, d)$. It is a metric invariant (it does not depend on the measure $\mu$ ).

The packing constant is often used in similar contexts (it is used extensively in the survey [Grigor'yan et al. 2004]). By the compactness of $M, C_{d}(M)$ is welldefined.

Note that $d$ is not necessarily the Riemannian distance. In fact, here are three typical situations in which it is easy to control the packing constant:

(I) $\left(M^{n}, g\right)$ is a closed Riemannian manifold and $d$ is the intrinsic distance on $M$ associated to the Riemannian metric $g$.

(II) $M^{n}$ is an immersed submanifold of another manifold $X$ (for example, hyperbolic or Euclidean space) and $d=d_{\mathrm{ext}}$ is the extrinsic distance, that is, the restriction to $M$ of the Riemannian distance on $X$.

(III) $M^{n}$ is a bounded domain with smooth boundary in a complete Riemannian manifold $X$ and again $d=d_{\text {ext }}$ is the extrinsic distance.

In the first case we can easily estimate the packing constant in terms of a lower bound of the Ricci curvature and the dimension, using the Bishop-Gromov inequality; see [Colbois and Maerten 2008, Example 2.1]. In cases (II) and (III), a simple argument shows that $C_{d}(M) \leq C_{d}(X)^{2}$, and so the packing constant of an immersed submanifold of Euclidean space (or of a manifold with nonnegative Ricci curvature) is bounded above by an absolute constant depending only on the dimension of $X$; in particular, it is independent on the Ricci curvature of $M$. For example, if $M$ is any submanifold of $\mathbb{R}^{m}$ then $C_{d}(M) \leq\left(1+3^{2 m}\right)^{2}$. Here $d$ is the extrinsic distance; for the intrinsic distance this is no longer true in general. 
1.2. Estimates for the Laplacian on functions. When the density $\sigma$ is positive, we can consider the following operator $L$ acting on any $u \in C^{\infty}(M)$ :

$$
L u=\Delta u-\frac{1}{\sigma}\langle\nabla u, \nabla \sigma\rangle .
$$

If $\partial M \neq \varnothing$, we assume Neumann boundary conditions. $L$ is self-adjoint when acting on $L^{2}(M, \mu)$, where $\mu=\sigma \mathrm{dvol}_{g}$, and is associated to the quadratic form

$$
u \mapsto \int_{M}|\nabla u|^{2} \sigma \mathrm{dvol}_{g} .
$$

The spectrum of $L$ is discrete and will be denoted by $\left\{\lambda_{k}(L)\right\}_{k=1}^{\infty}$. Note that $\lambda_{1}(L)=0$ and $\lambda_{2}(L)>0$. If $\sigma$ is constant (that is, $\mu$ is just a multiple of the Riemannian measure) one recovers the eigenvalues of the ordinary Laplacian on $M$. However, the generalization to Laplace-type operators will force us to consider nonconstant densities.

Theorem 1. Suppose $\mathcal{M}=(M, \mu, d)$ is a metric measured space as defined in Section 1.1 and assume that $\mu=\sigma \mathrm{dvol}_{g}$, with $\sigma>0$ everywhere on $M$. Let $L$ be the operator defined in (3). Then, for all $k \geq 1$, there exists a set $S$ of $k$ points $x_{1}, \ldots, x_{k} \in M$ such that

$$
r=8(k+1) C_{d}(M)^{2} \cdot \frac{\log \lambda_{k+1}(L)}{\sqrt{\lambda_{k+1}(L)}} \quad \text { implies } \quad \mu\left(S^{r}\right) \geq 1-r,
$$

provided that $\lambda_{k+1}(L) \geq$ e. Here $C_{d}(M)$ is the packing constant defined in (2).

Remarks. The estimate is sharp, in the sense that the decay $\log \lambda / \sqrt{\lambda}$ is optimal as $\lambda=\lambda_{k+1}(L)$ tends to infinity, and cannot be replaced by a function with a faster rate of decrease. We refer to Section A.2 for an explicit example.

If the eigenvalue $\lambda_{k+1}(L)$ is large (so that $r$ is small), then almost all of the measure $\mu$ is in the $r$-neighborhhood of $k$ suitable points: This is the concentration property that we want to emphasize.

There is an equivalent formulation of our estimate in terms of the so-called Lévy-Prokhorov distance between probability measures. If $(X, d)$ is a metric space, $\mathscr{B}(X)$ the borelian $\sigma$-algebra and $\mathscr{P}(X)$ the set of the probability measures on $X$, the Lévy-Prokhorov distance $d_{P}$ between two elements $v_{1}$ and $v_{2}$ of $\mathscr{P}(X)$ is defined as

$$
\begin{aligned}
& d_{P}\left(v_{1}, v_{2}\right) \\
& \quad=\inf \left\{r>0: v_{1}(C) \leq v_{2}\left(C^{r}\right)+r \text { and } v_{2}(C) \leq v_{1}\left(C^{r}\right)+r \text { for all } C \in \mathscr{B}(X)\right\} .
\end{aligned}
$$

See for example [Villani 2009, (6.5), page 97].

The following result is an equivalent formulation of Theorem 1. 
Theorem 2. In the hypothesis of Theorem 1, there exist $k$ points $x_{1}, \ldots, x_{k} \in M$ and weights $p_{1}, \ldots, p_{k} \in[0,1)$ such that $\sum p_{j}=1$ and

$$
d_{P}\left(\mu, \delta_{S}\right) \leq 8(k+1) C_{d}(M)^{2} \cdot \frac{\log \lambda_{k+1}(L)}{\sqrt{\lambda_{k+1}(L)}},
$$

where $\delta_{S}=\sum_{i=1}^{k} p_{i} \delta_{x_{i}}$ and $\delta_{x_{i}}$ is the Dirac measure concentrated at the point $x_{i}$.

In particular, for $k=1$ there exists a point $x_{1} \in M$ such that

$$
d_{P}\left(\mu, \delta_{x_{1}}\right) \leq 16 C_{d}(M)^{2} \cdot \frac{\log \lambda_{2}(L)}{\sqrt{\lambda_{2}(L)}} .
$$

The estimate is sharp: see Section A.2.

In other words, when the eigenvalue is large, the measure $\mu$ is close, in the Lévy-Prokhorov sense, to a weighted linear combination of the Dirac measures at the points $x_{1}, \ldots, x_{k}$.

The equivalence between the formulations in Theorem 1 and Theorem 2 will be proved in Section A.1.

Note that Theorems 1 and 2 apply obviously to the Laplacian acting on functions: it suffices to choose $\sigma=1 / \operatorname{Vol}(M)$. In that case the concentration is relative to the (normalized) Riemannian volume.

1.3. Estimates for vector bundle Laplacians. The next task will be to generalize Theorem 1 when the density $\sigma$ is only assumed to be nonnegative. For that purpose we introduce, in Section 2, a weaker notion of spectrum and prove the relevant Theorem 4. Besides being interesting in itself, Theorem 4 will lead to a concentration phenomenon of eigensections in the context of Laplacians acting on sections of a vector bundle.

So, consider a vector bundle $E$ over a compact Riemannian manifold $\left(M^{n}, g\right)$ with empty boundary, and denote by $\nabla$ a connection on $E$ that is compatible with the metric $g$ (see [Bérard 1988] for details). An operator $D$ acting on sections of the bundle is said to be of Laplace-type if it can be written $D=\nabla^{*} \nabla+T$, where $T$ is a symmetric endomorphism of the fiber. Then, $D$ is self-adjoint and elliptic. We list its eigenvalues as

$$
\lambda_{1}(D) \leq \lambda_{2}(D) \leq \cdots \leq \lambda_{k}(D) \leq \cdots
$$

and denote by $\left\{\psi_{1}, \psi_{2}, \ldots\right\}$ a corresponding orthonormal basis of eigensections.

Important examples of Laplace-type operators are given by the Laplacian acting on differential forms, by the square of the Dirac operator and by a Schrödinger operator acting on functions. In the first case, $T$ is the curvature term in the classical Bochner-Weitzenböck formula, in the second case it is multiplication by a constant multiple of the scalar curvature, and in the third case $T$ is just the potential. 
In the second main theorem we assume a large gap in the spectrum of $D$ and prove that eigensections concentrate their norms near a finite set of points.

Theorem 3. For each positive integer $k$ there is a set $S$ of $k$ points $x_{1}, \ldots, x_{k} \in M$ with the following property. Let $\psi$ be any unit $L^{2}$-norm linear combination of the first $k$ eigensections of $D$, and $\mu=|\psi|^{2} \mathrm{dvol}_{g}$. Then

$$
r=25 k\left(\frac{k^{2}(k+1) C_{d}(M)^{2}}{\lambda_{k+1}(D)-\lambda_{k}(D)}\right)^{1 / 3} \quad \text { implies } \quad \mu\left(S^{r}\right) \geq 1-r .
$$

Equivalently, the Lévy-Prokhorov distance between $\mu$ and a suitable linear combination of the Dirac measures at $x_{1}, \ldots, x_{k}$ is bounded above by $r$.

Example. We take $D$ to be the ordinary Laplacian on functions and assume that $\lambda_{k+1}$ tends to infinity while $\lambda_{k}$ is uniformly bounded. Then by Theorem 1 the Riemannian volume concentrates around $k$ suitable points $x_{1}, \ldots, x_{k}$. Theorem 3 then says that any eigenfunction associated to eigenvalues less than $\lambda_{k+1}$ will also concentrate its $L^{2}$-norm around $x_{1}, \ldots, x_{k}$.

Example. We take $D$ to be the Laplacian acting on $p$-forms and assume that the $p$-th Betti number of $M$ is positive, say $b_{p}(M)=k>0$. Then $\lambda_{k}(D)=0$ and $\lambda=\lambda_{k+1}(D)$ is the first positive eigenvalue of $D$. Assume that $\lambda$ is very large. Then the theorem gives the existence of $b_{p}(M)$ points such that all harmonic $p$-forms must concentrate their $L^{2}$-norms in a small neighborhood of the union of these points.

We also observe that, in general, a large gap in the spectrum of $D$ does not necessarily imply concentration of the Riemannian volume unless, of course, $D$ is the ordinary Laplacian, or there exist parallel sections (so that the density $\sigma=|\psi|^{2}$ is constant). We refer to Section A.3 for an explicit example.

The paper is structured as follows: In Section 2 we will prove Theorem 1 and a more general version of it, Theorem 4. In Section 3 we will establish the results for vector bundle Laplacians and prove Theorem 3. The appendix is devoted to the examples, in particular, the sharpness of the estimate given in Theorem 1 and 2.

\section{Estimates for functions}

2.1. A general estimate when the density is only nonnegative. We consider a compact manifold $M$ (with or without boundary) endowed with a distance function $d$ and a measure $\mu=\sigma \mathrm{dvol}_{g}$ as in Section 1.1. We first consider the general case in which $\sigma \geq 0$. This will be needed to treat Laplace-type operators, where the density $\sigma$ will be the squared norm of an eigensection, which can vanish at some points of $M$. However it is well known from elliptic theory that eigensections can vanish only on sets of measure zero. 
Let us then introduce the weak spectrum of the metric measured space $M=$ $(M, \mu, d)$ as follows. First, define the following Rayleigh quotient of the Lipschitz function $f$ (such that $\int_{M} f^{2} \mu>0$ ):

$$
R(f)=\int_{M}|\nabla f|^{2} \mu / \int_{M} f^{2} \mu .
$$

Denote by $W_{k}$ a vector space of Lipschitz functions on $M$ of finite dimension $k$. Then, for all integers $k \geq 0$ we define

$$
\lambda_{k+1}(M) \doteq \sup _{W_{k}} \inf \left\{R(f): f \perp W_{k}\right\} .
$$

It is clear that $\lambda_{1}(M)=0$. It is easy to check that the sequence $\lambda_{j}(M)$ is nondecreasing.

Having said that, we state the main theorem of this section.

Theorem 4. Let $M=(M, \mu, d)$ be as above, with $\mu=\sigma \mathrm{dvol}_{g}$ and $\sigma \geq 0$. Then, for each $k=1,2, \ldots$ we can find a set $S$ of $k$ points $x_{1}, \ldots, x_{k} \in M$ such that

$$
r=5\left(\frac{(k+1) C_{d}(M)^{2}}{\lambda_{k+1}(M)}\right)^{1 / 3} \quad \text { implies } \quad \mu\left(S^{r}\right) \geq 1-r .
$$

Remark. If the density $\sigma$ is strictly positive on $M$, then it is clear by the max-min principle that the weak spectrum of $M$ is equal to the spectrum of the self-adjoint elliptic operator $L$ acting on $L^{2}\left(M, \sigma \cdot \mathrm{dvol}_{g}\right)$ and already defined in (3). That is, $\lambda_{k}(M)=\lambda_{k}(L)$ for all $k$. In this case, using an upper bound of [Chung et al. 1997] and an additional measure theoretic lemma proved in [Colbois and Maerten 2008] we can prove Theorem 1, which is an improvement of Theorem 4 for large $\lambda=\lambda_{k+1}$ because $\log \lambda / \sqrt{\lambda}$ decays faster than $\lambda^{-1 / 3}$.

2.2. Preparatory results. In the next lemma we estimate the eigenvalues of $M$ as defined in the previous section. The first part follows from a standard argument involving plateau functions, which applies to our case. The second part is an estimate due to Chung, Grigor'yan and Yau.

Lemma 5. (a) Let $M=(M, \mu, d)$ and assume that $\mu=\sigma \cdot \mathrm{dvol}_{g}$ with $\sigma \geq 0$. Assume that there exist $k+1$ subsets of $M$, each of measure at least $\alpha>0$, which are $2 r$-separated (meaning that the distance between any two of the given sets is at least $2 r)$. Then $\lambda_{k+1}(\mathcal{M}) \leq 1 / \alpha r^{2}$.

(b) If the density $\sigma$ is strictly positive on $M$, then

$$
\lambda_{k+1}(M)=\lambda_{k+1}(L) \leq \log ^{2}(2 / \alpha) / r^{2},
$$

where $L$ is the operator $L u=\Delta u-\langle\nabla u, \nabla \sigma\rangle / \sigma$ defined in (3). 
Proof. (a) Fix a subspace $W$ of the space of Lipschitz functions on $M$, of finite dimension $k$. Let $A_{1}, \ldots, A_{k+1}$ be the subsets satisfying the assumptions, that is, $\int_{A_{j}} \mu=\int_{A_{j}} \sigma \mathrm{dvol}_{g} \geq \alpha$ and $d\left(A_{i}, A_{j}\right) \geq 2 r$ if $i \neq j$. For each $j=1, \ldots, k+1$, let $\phi_{j}$ be the plateau function

$$
\phi_{j}(x)= \begin{cases}1 & \text { on } A_{j}, \\ 1-d\left(x, A_{j}\right) / r & \text { on } \Omega_{j}=A_{j}^{r} \backslash A_{j}, \\ 0 & \text { on the complement of } A_{j}^{r} .\end{cases}
$$

Note that the $\phi_{j}$ are disjointly supported. Linear algebra shows that we can find numbers $a_{1}, \ldots, a_{k+1}$ such that the function $\phi=\sum_{j=1}^{k+1} a_{j} \phi_{j}$ is Lipschitz, $L^{2}(\mu)$ orthogonal to $W$ and nonzero. We can also assume that $\sum a_{j}^{2}=1$. The gradient of $\phi$ is supported on the union of the $\Omega_{j}$, and on $\Omega_{j}$ one has $|\nabla \phi| \leq\left|a_{j}\right| / r$ almost everywhere. Then

$$
\int_{M}|\nabla \phi|^{2} \mu \leq \frac{1}{r^{2}} \int_{M} \mu=\frac{1}{r^{2}}
$$

On the other hand,

$$
\int_{M} \phi^{2} \mu \geq \sum_{j} a_{j}^{2} \int_{A_{j}} \mu \geq \alpha .
$$

Therefore $R(\phi) \leq 1 /\left(\alpha r^{2}\right)$. Since $\phi$ was orthogonal to $W$, we get

$$
\inf \{R(f): f \perp W\} \leq 1 /\left(\alpha r^{2}\right) .
$$

The right side is independent of the subspace $W$; hence taking the supremum over all $k$-dimensional subspaces $W$ does not change the upper bound. Recalling the definition of $\lambda_{k+1}$, one obtains the first part of the lemma.

(b) If the density $\sigma$ is positive, we can use an estimate of Chung, Grigor'yan and Yau [1996]. It says that, if the subsets $A_{1}, \ldots, A_{k+1}$ are at distance at least $s$ from each other, then

$$
\lambda_{k+1}(L) \leq \frac{4}{s^{2}} \cdot \max _{i \neq j}\left(\log \frac{2}{\sqrt{\mu\left(A_{i}\right) \mu\left(A_{j}\right)}}\right)^{2} .
$$

The second inequality is now immediate by taking $s=2 r$.

We will use [Colbois and Maerten 2008, Corollary 2.3], which we state in a way more convenient to our purposes. Consider our metric space $(M, d)$ and recall the packing constant $C_{d}(M)$. Let $v$ be any measure on $M$.

Proposition 6. Let $N$ be a positive integer. Suppose that for a given $s>0$, we have for each $x \in M$

$$
v(B(x, s)) \leq \frac{v(M)}{4 C_{d}(M)^{2} N} .
$$

Then, there exist $N$ subsets $A_{1}, \ldots, A_{N}$ of $M$ such that $v\left(A_{i}\right) \geq v(M) /\left(2 C_{d}(M) N\right)$ for each $i$ and $d\left(A_{i}, A_{j}\right) \geq 3$ s for each $i \neq j$. 
We will use the proposition in the proof of Theorem 4 for $v$ given by the restriction of $\mu$ to a closed subset.

Proof of Theorem 4. Let $\lambda_{k+1}(\mathcal{M})=\lambda$ and assume that it is positive. Let

$$
r=5\left(\frac{(k+1) C_{d}(M)^{2}}{\lambda}\right)^{1 / 3}
$$

We will prove that there exist a set $S$ of suitably chosen points $x_{1}, \ldots, x_{k}$ (not necessarily distinct) such that

$$
\mu\left(S^{r}\right) \geq 1-r .
$$

We can suppose $r<1$.

Let $\alpha=r /\left(4(k+1) C_{d}(M)^{2}\right)$. By the definitions of $r$ and $\alpha$ one has

$$
\lambda=\frac{125}{4 \alpha r^{2}} \text {. }
$$

Step 1 (construction of the points). Choose $x_{1}$ so that $\mu\left(B\left(x_{1}, \frac{1}{4} r\right)\right) \geq \mu\left(B\left(x, \frac{1}{4} r\right)\right)$ for all $x \in M$, and set

$$
X_{1}=B\left(x_{1}, r\right)^{c} .
$$

Next, choose $x_{2} \in X_{1}$ so that $\mu\left(B\left(x_{2}, \frac{1}{4} r\right)\right) \geq \mu\left(B\left(x, \frac{1}{4} r\right)\right)$ for all $x \in X_{1}$, and set

$$
X_{2}=\left(B\left(x_{1}, r\right) \cup B\left(x_{2}, r\right)\right)^{c} .
$$

We continue in this way until we obtain $k$ points $x_{1}, \ldots, x_{k}$ : To construct the $j$-th point $x_{j} \in X_{j-1}$, we demand that $\mu\left(B\left(x_{j}, \frac{1}{4} r\right)\right) \geq \mu\left(B\left(x, \frac{1}{4} r\right)\right)$ for all $x \in X_{j-1}$ and define

$$
X_{j}=\left(B\left(x_{1}, r\right) \cup \cdots \cup B\left(x_{j}, r\right)\right)^{c} .
$$

Note that if $X_{j}$ is empty for some $j \leq k$, then $\mu\left(B\left(x_{1}, r\right) \cup \cdots \cup B\left(x_{j}, r\right)\right)=1>1-r$, so we can take $S=\left\{x_{1}, \ldots, x_{j-1}\right\}$. We have $\mu\left(S^{r}\right) \geq 1-r$ and the theorem is proved. So we can assume that

$$
X_{k}=\left(B\left(x_{1}, r\right) \cup \cdots \cup B\left(x_{k}, r\right)\right)^{c}
$$

is nonempty. Inequality (4) (and the theorem) follows if we show that $\mu\left(X_{k}\right) \leq r$. Step 2 (proof that $\mu\left(X_{k}\right) \leq r$ ). We argue by contradiction and show that the inequality

$$
\mu\left(X_{k}\right)>r
$$

cannot occur. Let us then assume (6) and denote by $B_{i}$ the ball $B\left(x_{i}, \frac{1}{4} r\right)$. By construction, the sets $B_{1}, \ldots, B_{k}$ and $X_{k}$ are $\frac{1}{2} r$-separated and

$$
\mu\left(B_{1}\right) \geq \mu\left(B_{2}\right) \geq \cdots \geq \mu\left(B_{k}\right) .
$$


First case. Assume $\mu\left(B_{k}\right) \geq \alpha$. Then $\mu\left(B_{j}\right) \geq \alpha$ for all $j$; moreover

$$
\mu\left(X_{k}\right) \geq r>\frac{r}{4(k+1) C_{d}(M)^{2}}=\alpha
$$

simply because $C_{d}(M) \geq 1$. Therefore the sets $B_{1}, \ldots, B_{k}, X_{k}$ are $\frac{1}{2} r$-separated and each of them has measure at least $\alpha$. By Lemma 5,

$$
\lambda=\lambda_{k+1}(M) \leq 16 /\left(\alpha r^{2}\right),
$$

which contradicts (5). Then the first case does not occur.

Second case. Assume $\mu\left(B_{k}\right)<\alpha$. Consider the closed subset $X=X_{k-1}$. By the definition of $x_{k}$, one has

$$
\mu\left(B\left(x, \frac{1}{4} r\right)\right) \leq \mu\left(B_{k}\right) \leq \alpha \quad \text { for all } x \in X .
$$

Recall that $X_{k} \subseteq X_{k-1}=X$.

We now consider the metric space $(M, d)$ with the measure $v$ given by the restriction of $\mu$ to the closed subspace $X$, that is, $v(A)=\mu(A \cap X)$. By (6) we have $r<\mu\left(X_{k}\right) \leq \mu(X)=v(M)$ and therefore

$$
v\left(B\left(x, \frac{1}{4} r\right) \leq \mu\left(B\left(x, \frac{1}{4} r\right) \leq \alpha=\frac{r}{4(k+1) C_{d}(M)^{2}} \leq \frac{v(M)}{4(k+1) C_{d}(M)^{2}} .\right.\right.
$$

By Proposition 6 applied for $s=\frac{1}{4} r$ and $N=k+1$, we conclude there exist $k+1$ subsets $A_{1}, \ldots, A_{k}$ that are $\frac{3}{4} r$-separated and satisfy

$$
\nu\left(A_{i}\right) \geq \frac{v(M)}{2 C_{d}(M)(k+1)}>\frac{r}{2 C_{d}(M)(k+1)} \geq 2 C_{d}(M) \alpha \geq 2 \alpha \quad \text { for all } i .
$$

Then $\mu\left(A_{i}\right) \geq 2 \alpha$ for all $i$. Applying Lemma 5, one would obtain

$$
\lambda=\lambda_{k+1}(M) \leq \frac{32}{9 \alpha r^{2}},
$$

which again contradicts (5). The proof of Theorem 4 is now complete.

Proof of Theorem 1. Set $\lambda_{k+1}(\mathcal{M})=\lambda$ and assume $\lambda \geq e$. Let

$$
r=\frac{\beta \log \lambda}{\sqrt{\lambda}},
$$

where $\beta=8(k+1) C_{d}(M)^{2}$. We will find a set $S$ of $k$ points $x_{1}, \ldots, x_{k}$ such that

$$
\mu\left(S^{r}\right) \geq 1-r,
$$

which is the statement of the theorem.

Set $\alpha=r /\left(4(k+1) C_{d}(M)^{2}\right)$. We first observe that

$$
\lambda>\frac{256}{r^{2}} \log ^{2}(2 / \alpha) \text {. }
$$


In fact (9) gives $\lambda=\beta^{2} \log ^{2} \lambda^{2} / r^{2} \geq \beta^{2} / r^{2}$, and substituting inside $\log \lambda$ we get (11) because $\beta / r=2 / \alpha$ by the definitions of $\alpha$ and $\beta$ and the fact that $\beta \geq 8$.

To show (10) we follow Step 1 and Step 2 exactly as in the proof of the previous theorem: We construct the points $x_{1}, \ldots, x_{k}$ as before and show that the inequality $\mu\left(X_{k}\right)>r$ leads to a contradiction with the inequality (11). The only change is to use the second inequality of Lemma 5 instead of the first, so that (7) and (8) respectively become

$$
\lambda \leq \frac{16}{r^{2}} \log ^{2}(2 / \alpha) \quad \text { and } \quad \lambda \leq \frac{64}{9 r^{2}} \log ^{2}(2 / \alpha),
$$

both of which contradict (11).

Remark. It is not possible to replace the constant $\beta$ in (9) by $\beta(\lambda)$ for a function $\beta(\lambda) \rightarrow 0$ as $\lambda \rightarrow \infty$. In fact, taking $\beta=$ constant is the optimal choice for the radius $r$; see Section A.2.

\section{The estimate for Laplace-type operators}

In this section we prove Theorem 3 .

Theorem 7. Let $M^{n}$ be a compact Riemannian manifold without boundary and D any Laplace-type operator on M. Fix integers $i$ and $k$ with $i \leq k$ and consider the $m$-m-space $\left(M, \mu_{i}, d\right)$, where $\mu_{i}=\left|\psi_{i}\right|^{2} \cdot \mathrm{dvol}_{g}$ and $\psi_{i}$ is a unit norm eigensection associated to $\lambda_{i}(D)$. Then there exists a set $S_{i}$ of $k$ points $x_{1}^{i}, \ldots, x_{k}^{i} \in M$ such that

$$
r=5\left(\frac{k(k+1) C_{d}(M)^{2}}{\lambda_{k+1}(D)-\lambda_{i}(D)}\right)^{1 / 3} \quad \text { implies } \quad \mu_{i}\left(S_{i}^{r}\right) \geq 1-r .
$$

Of course, the result is significant only when the gap $\lambda_{k+1}(D)-\lambda_{i}(D)$ is large enough. As the gap $\lambda_{k+1}(D)-\lambda_{k}(D)$ increases to $\infty$, we see that any eigensection associated to $\lambda_{i}(D)$, with $i \leq k$, tends to concentrate its norm around at most $k$ points $x_{1}^{i}, \ldots, x_{k}^{i}$, a priori depending on $i$. It is natural to ask if there is a relation between all these points for different eigenvalues. We can in fact show that, as the gap tends to infinity, all squared norms $\left|\psi_{1}\right|^{2}, \ldots,\left|\psi_{k}\right|^{2}$ will concentrate around a common set of $k$ points. Actually, we will show that this also happens for the squared norm of any section in the direct sum of the first $k$ eigenspaces; this is the statement of Theorem 3 .

Proof of Theorem 7. The proof depends on the following two lemmas, in which we bound the gaps in the spectrum of $D$ by the weak spectrum of the m-m-spaces $\mu$ corresponding to the densities $\sigma=|\psi|^{2}$, where $\psi$ is an eigensection of $D$. We then apply Theorem 4 to conclude.

Recall that $D=\nabla^{*} \nabla+T$, where $T$ is a symmetric endomorphism of the fiber. 
So the quadratic form associated to $D$ is

$$
2(\psi)=\int_{M}|\nabla \psi|^{2}+\langle T \psi, \psi\rangle,
$$

which is defined on the space of $H^{1}$-sections of the bundle (here integration is with respect to the Riemannian measure $\mathrm{dvol}_{g}$ ). We fix an orthonormal basis of eigensections of $D$ and denote it by $\left(\psi_{1}, \psi_{2}, \ldots\right)$.

Lemma 8. Let $f$ be a Lipschitz function on $M$ and $\psi$ a smooth section of the bundle. Then

$$
2(f \psi)=\int_{M} f^{2}\langle D \psi, \psi\rangle+|\nabla f|^{2}|\psi|^{2} .
$$

Lemma 9. Fix a positive integer $k$ and let $i \leq k$. Let $\psi_{i}$ be an eigensection associated to $\lambda_{i}(D)$, of unit $L^{2}$-norm, and consider the m-m-space $\mu_{i}=\left(M, \mu_{i}, d\right)$ where $\mu_{i}=\left|\psi_{i}\right|^{2} \mathrm{dvol}_{g}$. Then

$$
\lambda_{k+1}(D)-\lambda_{i}(D) \leq k \lambda_{k+1}\left(M_{i}\right) .
$$

Theorem 7 now follows immediately from Lemma 9 and Theorem 4 applied with the density $\sigma=\left|\psi_{i}\right|^{2}$.

Proof of Lemma 8. On the subset where $\nabla f$ exists (hence almost everywhere on $M$ ), one has

$$
|\nabla(f \psi)|^{2}=|\nabla f|^{2}|\psi|^{2}+f^{2}|\nabla \psi|^{2}+2 f\left\langle\nabla_{\nabla f} \psi, \psi\right\rangle .
$$

Now

$$
\int_{M} 2 f\left\langle\nabla_{\nabla f} \psi, \psi\right\rangle=\int_{M} \frac{1}{2}\left\langle\nabla f^{2}, \nabla|\psi|^{2}\right\rangle=\int_{M} \frac{1}{2} f^{2} \Delta|\psi|^{2},
$$

and hence

$$
\begin{aligned}
2(f \psi) & =\int_{M}|\nabla(f \psi)|^{2}+\langle T(f \psi), f \psi\rangle \\
& =\int_{M} f^{2}\left(|\nabla \psi|^{2}+\frac{1}{2} \Delta|\psi|^{2}+\langle T \psi, \psi\rangle\right)+|\nabla f|^{2}|\psi|^{2} .
\end{aligned}
$$

Now recall the identity (Bochner formula) $\langle D \psi, \psi\rangle=|\nabla \psi|^{2}+\frac{1}{2} \Delta|\psi|^{2}+\langle T \psi, \psi\rangle$. The lemma follows.

Proof of Lemma 9. Given the metric-measure space $M=(M, \mu, d)$, recall the definition of weak spectrum:

$$
\lambda_{h+1}(M)=\sup _{W_{h}} \inf \left\{R(f): f \perp W_{h}\right\}, \quad \text { where } R(f)=\int_{M}|\nabla f|^{2} \mu / \int_{M} f^{2} \mu,
$$

and $W_{h}$ denotes a vector subspace of Lipschitz functions having dimension $h$. We will write for brevity $\lambda_{i}(\mathcal{M})=\lambda_{i}$. 
Fix $\epsilon>0$. Then, for all integers $k \in \mathbb{N}$ we construct a $(k+1)$-dimensional subspace $W_{k+1}$ of the space of Lipschitz functions on $M$ such that, for all $f \in W_{k+1}$,

$$
R(f) \leq k\left(\lambda_{k+1}+\epsilon\right) .
$$

Set $W_{1}=\operatorname{span}\left(f_{1}\right)$, where $f_{1}$ is the constant function 1 . By definition, there exists a nonvanishing smooth function $f_{2}$ that is orthogonal to $W_{1}$ and satisfies

$$
R\left(f_{2}\right) \leq \lambda_{2}+\epsilon .
$$

Set $W_{2}=\operatorname{span}\left(f_{1}, f_{2}\right)$. We can assume that $f_{2}$ has unit $L^{2}$-norm. Continuing this process, we get $W_{k+1}=\operatorname{span}\left(f_{1}, \ldots, f_{k+1}\right)$, where $\left(f_{1}, \ldots, f_{k+1}\right)$ is an orthonormal set and, for all $j=1, \ldots, k+1$,

$$
R\left(f_{j}\right) \leq \lambda_{j}+\epsilon \leq \lambda_{k+1}+\epsilon .
$$

Let us prove (12). Let $f=\sum_{i=1}^{k+1} a_{i} f_{i}$ be a function in $W_{k+1}$. We can assume that it has unit norm, so that $\sum_{i} a_{i}^{2}=1$. By the triangle inequality, since $\nabla f_{1}=0$, one has $|\nabla f| \leq \sum_{i=2}^{k+1}\left|a_{i}\right|\left|\nabla f_{i}\right|$. By the Schwarz inequality, $|\nabla f|^{2} \leq \sum_{i=2}^{k+1}\left|\nabla f_{i}\right|^{2}$ and therefore, by (13),

$$
R(f) \leq \sum_{i=2}^{k+1} R\left(f_{i}\right) \leq k\left(\lambda_{k+1}+\epsilon\right) .
$$

We can now prove the lemma. Fix $\epsilon>0$ and consider the m-m-space $M_{i}$ with measure $\mu_{i}=\left|\psi_{i}\right|^{2} \mathrm{dvol}_{g}$, as in the statement of the lemma. Let $W_{k+1}$ be the subspace satisfying (12). By linear algebra, we can find a nonvanishing $f \in W_{k+1}$ such that the section $f \psi_{i}$ has unit norm and is orthogonal to the first $k$ eigensections $\psi_{1}, \ldots, \psi_{k}$ of the spectrum of $D$. Using $f \psi_{i}$ as test-section for the eigenvalue $\lambda_{k+1}(D)$, we obtain by Lemma 8

$$
\lambda_{k+1}(D) \leq 2\left(f \psi_{i}\right)=\int_{M} f^{2}\left\langle D \psi_{i}, \psi_{i}\right\rangle+|\nabla f|^{2}\left|\psi_{i}\right|^{2} .
$$

Since $\left\langle D \psi_{i}, \psi_{i}\right\rangle=\lambda_{i}(D)\left|\psi_{i}\right|^{2}$, this becomes

$$
\lambda_{k+1}(D)-\lambda_{i}(D) \leq R(f) \leq k\left(\lambda_{k+1}\left(M_{i}\right)+\epsilon\right),
$$

by (12). Letting $\epsilon \rightarrow 0$ we obtain the assertion.

Proof of Theorem 3. Let us start with the formal proof by considering an orthonormal basis $\left(\psi_{1}, \ldots, \psi_{k}\right)$ of the direct sum of the first $k$ eigenspaces of $D$. Given $\mu_{j}=\left|\psi_{j}\right|^{2} \cdot \mathrm{dvol}_{g}$, let us introduce the following auxiliary measure, which is just the average of the $\mu_{j}$ :

$$
\tilde{\mu}=\frac{1}{k} \sum_{j=1}^{k} \mu_{j} .
$$


We also fix the radius

$$
r=5\left(\frac{k^{2}(k+1) C_{d}(M)^{2}}{\lambda_{k+1}(D)-\lambda_{k}(D)}\right)^{1 / 3} .
$$

The theorem follows from two claims.

Claim 1. There exists a set of points $Q=\left\{y_{1}, \ldots, y_{l}\right\}$ with the property that

$$
\tilde{\mu}\left(B\left(y_{j}, r\right)\right) \geq r / k^{2} \text { for all } j \text { and } \tilde{\mu}\left(Q^{r}\right) \geq 1-2 r .
$$

Claim 2. There exists a subset $T=\left\{x_{1}, \ldots, x_{m}\right\}$ of $Q$, with $m \leq k$, such that

$$
\tilde{\mu}\left(T^{5 r}\right) \geq 1-5 r .
$$

(This gives a concentration result for the averaged measure $\tilde{\mu}$ ).

Thanks to Claims 1 and 2, we can conclude as follows. Let $\psi=\sum_{i=1}^{k} a_{i} \psi_{i}$ be any unit norm section in the direct sum of the first $k$ eigenspaces of $D$ (so that $\sum_{i} a_{i}^{2}=1$ ), and let $\mu=|\psi|^{2} \mathrm{dvol}_{g}$. By the Schwarz inequality we have, at any point,

$$
|\psi|^{2} \leq\left(\sum_{i}\left|a_{i}\right|\left|\psi_{i}\right|\right)^{2} \leq \sum_{i}\left|\psi_{i}\right|^{2}
$$

that is, $\mu \leq k \tilde{\mu}$. We deduce $\mu\left(\left(T^{5 k r}\right)^{c}\right) \leq \mu\left(\left(T^{5 r}\right)^{c}\right) \leq k \tilde{\mu}\left(\left(T^{5 r}\right)^{c} \leq 5 k r\right.$ by Claim 2 . We now take $S=T$. Then $\mu\left(S^{5 k r}\right) \geq 1-5 k r$ and the theorem follows.

For the proof of the two claims we need a lemma. We can assume $r<1 / 5$.

Lemma 10. Assume there exist $k+1$ subsets $A_{1}, \ldots, A_{k+1}$ that are $2 r$-separated and have $\tilde{\mu}$-measure at least $\beta$. Then

$$
\lambda_{k+1}(D)-\lambda_{k}(D) \leq \frac{k}{\beta r^{2}} .
$$

Proof. As in the proof of Lemma 5, we can construct $k+1$ disjointly supported, plateau functions $f_{1}, \ldots, f_{k+1}$ with $R_{\tilde{\mu}}\left(f_{j}\right) \leq 1 /\left(\beta r^{2}\right)$ for each $j$, where $R_{\tilde{\mu}}$ is the Rayleigh quotient relative to the measure $\tilde{\mu}$. Since $\tilde{\mu}$ is the average of the $\mu_{j}$, we see that for any nonnegative function $f$ there is an index $i$ (depending on $f$ ) such that $\int_{M} f \tilde{\mu} \leq \int_{M} f \mu_{i}$. Therefore, for each $j=1, \ldots, k+1$ there is an index $\alpha(j)=1, \ldots, k$ such that

$$
R_{\tilde{\mu}}\left(f_{j}\right)=\frac{\int_{M}\left|\nabla f_{j}\right|^{2} \tilde{\mu}}{\int_{M} f_{j}^{2} \tilde{\mu}} \geq \frac{1}{k} \frac{\int_{M}\left|\nabla f_{j}\right|^{2} \mu_{\alpha(j)}}{\int_{M} f_{j}^{2} \mu_{\alpha(j)}} \geq \frac{1}{k} R_{\mu_{\alpha(j)}}\left(f_{j}\right)
$$

and then $R_{\mu_{\alpha(j)}}\left(f_{j}\right) \leq k /\left(\beta r^{2}\right)$ for all $j$. We consider the sections $s_{j}=f_{j} \psi_{\alpha(j)}$ for $j=1, \ldots, k+1$; they are disjointly supported and we can use them as test-sections for the eigenvalue $\lambda_{k+1}(D)$. Using Lemma 8 one sees that

$$
\lambda_{k+1}(D)-\lambda_{k}(D) \leq \sup _{j}\left\{R_{\mu_{\alpha(j)}}\left(f_{j}\right)\right\} \leq k /\left(\beta r^{2}\right) .
$$


Proof of Claim 1. For all $j \leq k$ we observe from (14) that

$$
r \geq 5\left(\frac{k(k+1) C_{d}(M)^{2}}{\lambda_{k+1}(D)-\lambda_{j}(D)}\right)^{1 / 3} .
$$

So, by Theorem 7, there exist finite subsets $S_{1}, \ldots, S_{k} \subseteq M$ of cardinality less than or equal to $k$ such that $\mu_{j}\left(S_{j}^{r}\right) \geq 1-r$ for all $j$. We set $P=S_{1} \cup \cdots \cup S_{k}$ and observe that, by the definition of $\tilde{\mu}$,

$$
\tilde{\mu}\left(P^{r}\right) \geq 1-r .
$$

We now consider the subset $Q=\left\{y_{1}, \ldots, y_{l}\right\}$ formed by all points $y_{j} \in P$ such that $\tilde{\mu}\left(B\left(y_{j}, r\right)\right) \geq r / k^{2}$. Let $Q^{\prime}=P \backslash Q$. Then by definition $\tilde{\mu}\left(\left(Q^{\prime}\right)^{r}\right) \leq r$. Since $\tilde{\mu}\left(\left(Q^{\prime}\right)^{r}\right)+\tilde{\mu}\left(Q^{r}\right) \geq 1-r$ by $(15)$, we obtain

$$
\tilde{\mu}\left(Q^{r}\right) \geq 1-2 r
$$

as claimed. Note that $Q$ is not empty because $r<1 / 5$ by assumption.

Proof of Claim 2. We construct the subset $T=\left\{x_{1}, \ldots, x_{m}\right\}$ of $Q$ as follows. Set $x_{1}=y_{1}$. If there exists some point $y_{j} \in Q$ in the complement of $B\left(x_{1}, 4 r\right)$, we select it and denote it by $x_{2}$. Next, if there exists a point of $Q$ in the complement of $B\left(x_{1}, 4 r\right) \cup B\left(x_{2}, 4 r\right)$, we select it and denote it by $x_{3}$, and so on. We iterate the process until it is possible, and obtain after $m \leq l$ steps the required subset $T$.

Assume that $m \geq k+1$. Then the balls $A_{j}=B\left(x_{j}, r\right)$ with $j=1, \ldots, k+1$ are $2 r$-separated by construction, and have $\tilde{\mu}$-measure at least equal to $\beta=r / k^{2}$. By Lemma 10 we see that

$$
\lambda_{k+1}(D)-\lambda_{k}(D) \leq k^{3} / r^{3} .
$$

However, the definition (14) of $r$ gives $\lambda_{k+1}(D)-\lambda_{k}(D)=c / r^{3}$ with the constant $c=125 k^{2}(k+1) C_{d}(M)^{2}>k^{3}$ and we get a contradiction with (17).

Therefore $m \leq k$.

By the construction of $T$, every point $y_{j} \in Q$ is at distance not greater than $4 r$ to some point of $T$, that is, $Q \subseteq T^{4 r}$. By the triangle inequality $Q^{r} \subseteq T^{5 r}$ and therefore, by (16)

$$
\tilde{\mu}\left(T^{5 r}\right) \geq \tilde{\mu}\left(Q^{r}\right) \geq 1-2 r>1-5 r,
$$

and Claim 2 follows.

\section{Appendix}

A.1. Facts about the Lévy-Prokhorov distance. Recall that the Lévy-Prokhorov distance $d_{P}$ between two probability measures defined on the same metric space $(M, d)$ is

$$
d_{P}\left(v_{1}, v_{2}\right)=\inf \left\{r>0: v_{1}(C) \leq v_{2}\left(C^{r}\right)+r \text { and } v_{2}(C) \leq v_{1}\left(C^{r}\right)+r \text { for all } C\right\} .
$$


Proposition 11. Let $(M, \mu, d)$ be an $m$-m-space, and let $S=\left\{x_{1}, \ldots, x_{k}\right\}$ be a set of $k$ points in $M$ and $r>0$. Then $\mu\left(S^{r}\right) \geq 1-r$ if and only if there exist weights $p_{1}, \ldots, p_{k} \in[0,1)$ such that $\sum p_{j}=1$ and $d_{P}(\mu, \delta) \leq r$, where $\delta=\sum_{i=1}^{k} p_{i} \delta_{x_{i}}$ and $\delta_{x_{i}}$ is the Dirac measure concentrated at the point $x_{i}$.

Proof. Suppose first that $d_{P}(\mu, \delta) \leq r$. Then, choosing $C=S$ in the definition of $d_{P}$, we have $1=\delta(S) \leq \mu\left(S^{r}\right)+r$ and therefore $\mu\left(S^{r}\right) \geq 1-r$.

To prove the converse, we assume $\mu\left(S^{r}\right) \geq 1-r$. We first define the weights $p_{i}$. Denote by $B_{i}$ the ball $B\left(x_{i}, r\right)$ and consider the sets $\left\{A_{i}\right\}_{i=1}^{k}$ defined by

$$
\left\{\begin{array}{l}
A_{1}=B_{1}, \\
A_{i}=B_{i} \cap\left(B_{1} \cup \cdots \cup B_{i-1}\right)^{c} \quad \text { for } i \geq 2 .
\end{array}\right.
$$

Then $A_{i} \subseteq B_{i}$ and $A_{i} \cap A_{j}=\varnothing$ if $i \neq j$. Set $A=A_{1} \cup \cdots \cup A_{k}$. Then $A=$ $B_{1} \cup \cdots \cup B_{k}=S^{r}$, so that $\mu(A)=\mu\left(S^{r}\right) \geq 1-r$.

We now choose the weights $p_{i}=\mu\left(A_{i}\right) / \mu(A)$.

The proof is complete if we show that, for each Borel subset $C$, we have

$$
\left\{\begin{array}{l}
\delta(C) \leq \mu\left(C^{r}\right)+r \\
\mu(C) \leq \delta\left(C^{r}\right)+r .
\end{array}\right.
$$

We can order the points so that $x_{1}, \ldots, x_{t} \in C$ and $x_{j} \notin C$ for $j=t+1, \ldots, k$. Then $\delta(C)=p_{1}+\cdots+p_{t}$. Now $B_{1} \cup \cdots \cup B_{t} \subseteq C^{r}$; since $A_{i} \subseteq B_{i}$ and the $A_{i}$ are pairwise disjoint, we have

$$
\mu\left(A_{1}\right)+\cdots+\mu\left(A_{t}\right) \leq \mu\left(B_{1} \cup \cdots \cup B_{t}\right) \leq \mu\left(C^{r}\right) .
$$

Then

$$
\begin{aligned}
\delta(C)=p_{1}+\cdots+p_{t} & =\frac{\mu\left(A_{1}\right)+\cdots+\mu\left(A_{t}\right)}{\mu(A)} \\
& =\mu\left(A_{1}\right)+\cdots+\mu\left(A_{t}\right)+\frac{\mu\left(A_{1}\right)+\cdots+\mu\left(A_{t}\right)}{\mu(A)}(1-\mu(A)) \\
& \leq \mu\left(C^{r}\right)+1-\mu(A) \\
& \leq \mu\left(C^{r}\right)+r,
\end{aligned}
$$

which proves the first inequality in (18).

For the second, write

$$
\mu(C)=\mu\left(C \cap A_{1}\right)+\cdots+\mu\left(C \cap A_{k}\right)+\mu\left(C \cap A^{c}\right)
$$

and note that $x_{i} \in C^{r}$ if $C \cap A_{i} \neq \varnothing$. Since $\mu\left(C \cap A_{i}\right) \leq \mu\left(A_{i}\right)=p_{i} \mu(A) \leq p_{i}$ and $\mu\left(C \cap A^{c}\right) \leq \mu\left(A^{c}\right) \leq r$, we have

$$
\mu(C) \leq \sum_{i: x_{i} \in C^{r}} p_{i}+r \leq \delta\left(C^{r}\right)+r .
$$


A.2. Theorem 1 is sharp. For $R>0$, let $M_{R}$ be the surface of revolution in $\mathbb{R}^{3}$ :

$$
M_{R}=\left\{(x, y, z) \in \mathbb{R}^{3}: y^{2}+z^{2}=e^{-2 R x} / R^{2}, x \in[0,1]\right\},
$$

and consider the metric measure space $\left(M_{R}, \mu, d\right)$, where $\mu$ is the normalized Riemannian measure and $d$ is the extrinsic distance inherited from $\mathbb{R}^{3}$. By a calculation in [Friedman and Tillich 2000], one knows that

$$
\lambda_{2}\left(M_{R}\right) \geq \frac{1}{8} R^{2}
$$

(we take the Neumann boundary conditions). By the equivalent formulation of Theorem 1, given in Theorem 2, for each $R$ there exists a point $p \in M_{R}$ such that

$$
d_{P}\left(\mu, \delta_{p}\right) \leq \gamma_{R} \frac{\log \lambda_{R}}{\sqrt{\lambda_{R}}}
$$

for the constant $\gamma_{R}=16 C_{d}\left(M_{R}\right)^{2}$, where we set $\lambda_{R}=\lambda_{2}\left(M_{R}\right)$. However, since we use the extrinsic distance, the constant $\gamma_{R}$ admits a uniform upper bound by the packing constant of $\mathbb{R}^{3}$ (see Section 1.1); hence

$$
d_{P}\left(\mu, \delta_{p}\right) \leq \gamma \frac{\log \lambda_{R}}{\sqrt{\lambda}_{R}}
$$

for some $p \in M_{R}$ and an absolute constant $\gamma$ (we can take in fact $\gamma=16\left(1+3^{6}\right)^{2}$ ).

Now, when $R$ goes to $\infty$ the first positive eigenvalue $\lambda_{R}$ goes to $\infty$ by (19). Therefore, by (20), the normalized Riemannian measure $\mu$ concentrates at some point of $M_{R}$ : This is quite evident and can be verified directly from the definition of $M_{R}$, because the limit metric measure space as $R \rightarrow \infty$ (in any reasonable sense) is the unit interval $[0,1]$ endowed with its canonical distance and the Dirac measure supported at 0 . In fact, one can check that the relative measure of a set at positive distance $\alpha$ from the circle $\{x=0\}$ decreases to zero like $e^{-\alpha R}$.

In this section we show that, apart from the constant $\gamma$, the inequality (20) is actually sharp.

Theorem 12. Let $M_{R}$ and $\lambda_{R}$ be as above. Then there exists $R_{0}$ such that, for all $R \geq R_{0}$ and for all $q \in M_{R}$, one has

$$
d_{P}\left(\mu, \delta_{q}\right) \geq \frac{1}{48} \frac{\log \lambda_{R}}{\sqrt{\lambda_{R}}} .
$$

Lemma 13. Assume that there exist two subsets $A$ and $B$ with relative volume at least $s$, and such that $d(A, B) \geq 2$ s. Then $d_{P}\left(\mu, \delta_{q}\right) \geq$ s for all $q \in M_{R}$.

Proof. Assume that there exists $q \in M_{R}$ such that $d_{P}\left(\mu, \delta_{q}\right)<s$. One sees from the definition of $d_{P}$ that $\mu(B(q, s))>1-s$ and therefore $\mu(B(q, s))+\mu(A)>1$. So $A$ must intersect $B(q, s)$ and there exists $a \in A$ such that $d(a, q)<s$. Similarly, 
there exists $b \in B$ with $d(b, q)<s$. Applying the triangle inequality we get a contradiction with the assumption $d(A, B) \geq 2 s$.

Proof of Theorem 12. By (19) one has $\lambda_{R}>\frac{1}{9} R^{2}$; hence, for $R$ large,

$$
\frac{1}{48} \frac{\log \lambda_{R}}{\sqrt{\lambda_{R}}} \leq \frac{1}{8} \frac{\log R}{R} .
$$

So, it is enough to show that

$$
d_{P}\left(\mu, \delta_{q}\right) \geq \frac{1}{8} \frac{\log R}{R} \text { for } R \text { large and for all } q \in M_{R} .
$$

For $L<L^{\prime}$ in the interval $[0,1]$, consider the strip

$$
M_{\left[L, L^{\prime}\right]}=\left\{(x, y, z) \in M_{R}: L \leq x \leq L^{\prime}\right\} .
$$

We will apply the lemma, taking

$$
A=M_{[0,1 / R]}, \quad B=M_{[(1 / 2)(\log R) / R, 1]}, \quad s=\frac{1}{8}(\log R) / R .
$$

We need the simple volume estimate

$$
\mu\left(M_{\left[L, L^{\prime}\right]}\right) \geq \frac{e^{-L R}-e^{-L^{\prime} R}}{2\left(1-e^{-R}\right)} .
$$

In fact, observe that $M_{R}$ is obtained by rotating the curve $y=e^{-R x} / R$ around the $x$-axis. Then

$$
\operatorname{Vol}\left(M_{\left[L, L^{\prime}\right]}\right)=\frac{2 \pi}{R} \int_{L}^{L^{\prime}} e^{-R x} d s, \quad \text { with } d s=\sqrt{1+e^{-2 R x}} d x .
$$

Inequality (21) now follows from observing that $d x \leq d s<2 d x$ and recalling that $\mu\left(M_{\left[L, L^{\prime}\right]}\right)=\operatorname{Vol}\left(M_{\left[L, L^{\prime}\right]}\right) / \operatorname{Vol}\left(M_{[0,1]}\right)$.

By the volume estimate in (21),

$$
\mu(A) \geq \frac{1-e^{-1}}{2\left(1-e^{-R}\right)}, \quad \mu(B) \geq \frac{R^{-1 / 2}-e^{-R}}{2\left(1-e^{-R}\right)}, \quad d(A, B) \geq \frac{1}{2} \frac{\log R}{R}-\frac{1}{R} .
$$

It is now clear that, for $R \geq R_{0}$ sufficiently large, one has $\mu(A) \geq s, \mu(B) \geq s$ and $d(A, B) \geq 2 s$. The lemma gives $d_{P}\left(\mu, \delta_{q}\right) \geq s=\frac{1}{8}(\log R) / R$ and the theorem is proved.

A.3. Example for differential forms. We will now construct an example with a large gap on the spectrum of the Laplacian on $p$-forms, but in which there is no concentration of the Riemannian volume.

Indeed, the construction of large eigenvalues for $p$-forms is well known; see [Gentile and Pagliara 1995; Guerini 2004; Colbois and El Soufi 2006]. We can 
easily adapt the construction of Gentile and Pagliara for an hypersurface in $\mathbb{R}^{n+1}$, and we will only briefly sketch it.

We begin with a hypersurface $M_{0} \subset \mathbb{R}^{n+1}$, with $p$-th De Rham cohomology space of a given positive dimension. Then we deform $M_{0}$ by adding a long cylinder $[0, L] \times S^{n-1}$ closed by a hemisphere. We denote by $M_{L}$ this family of manifolds, whose volume is of the order of $L$ as $L \rightarrow \infty$. Gentile and Pagliara showed that, for $2 \leq p \leq n-2$, the nonzero $p$-forms spectrum of $M_{L}$ is bounded below by a positive constant $C$ not depending on $L$.

After renormalisation by a factor of order $L^{-1 / n}$, we get a family of constant volume 1 , with first nonzero eigenvalue for $p$-forms going to $\infty$ with $L$. Using the extrinsic Euclidean distance, we see that the packing constant is uniformly bounded, and we can conclude that the $L^{2}$-norms of the harmonic forms have to concentrate, indeed on the part corresponding to $M_{0}$.

However, there is no concentration of the volume; the part $M_{0}$ concentrates to a point and the cylinder looks like a homogeneous 1-dimensional cylinder of length $L^{1-1 / n}$.

A.4. Expanders. In this section we construct a family of manifolds $\bar{M}_{i}$ of fixed dimension $n$ such that $\lambda_{2}\left(\bar{M}_{i}\right) \rightarrow \infty$ but for which there is no concentration of the volume around any point.

We start from an $n$-dimensional compact, hyperbolic manifold $M_{i}$ such that $\operatorname{Vol}\left(M_{i}\right) \rightarrow \infty$ as $i \rightarrow \infty$ and $\lambda_{2}\left(M_{i}\right) \geq C(n)>0$, where $C(n)$ is a constant not depending on $i$. Such examples do exist (see for example [Brooks 1986]), even if their construction, related to the concept of expanders, is not easy. The $M_{i}$ can be realized as coverings of a fixed manifold. The diameter of $M_{i}$ is proportional to $\ln \operatorname{Vol}\left(M_{i}\right)$, and hence tends to infinity as $i \rightarrow \infty$.

So, if we multiply the metric of $M_{i}$ by $\left(\operatorname{diam}\left(M_{i}\right)\right)^{-1}$, and denote by $\bar{M}_{i}$ the new family of Riemannian manifolds, it is clear that $\lambda_{2}\left(\bar{M}_{i}\right) \rightarrow \infty$ but diam $\bar{M}_{i}=1$. Since $\bar{M}_{i}$ is a covering, the distribution of the volume is uniform, and we see that it cannot concentrate in a neighborhood of a single point. It concentrates however in the sense described in [Chung et al. 1996]: Two sets $A_{i}, B_{i} \subset \bar{M}_{i}$ of volume no less than $\kappa \operatorname{Vol}\left(\bar{M}_{i}\right)$ (with a fixed $\kappa>0$ ) have to be very close to each other, even if $\kappa$ is small.

\section{References}

[Bérard 1988] P. H. Bérard, "From vanishing theorems to estimating theorems: the Bochner technique revisited”, Bull. Amer. Math. Soc. (N.S.) 19 (1988), 371-406. MR 89i:58152 Zbl 0662.53037

[Brooks 1986] R. Brooks, "The spectral geometry of a tower of coverings", J. Differential Geom. 23:1 (1986), 97-107. MR 87j:58095 Zbl 0576.58033

[Cheng 1975] S. Y. Cheng, "Eigenvalue comparison theorems and its geometric applications", Math. Z. 143:3 (1975), 289-297. MR 51 \#14170 Zbl 0329.53035 
[Chung et al. 1996] F. R. K. Chung, A. Grigor'yan, and S.-T. Yau, "Upper bounds for eigenvalues of the discrete and continuous Laplace operators", Adv. Math. 117:2 (1996), 165-178. MR 96m:58254 Zbl 0844.53029

[Chung et al. 1997] F. R. K. Chung, A. Grigor'yan, and S.-T. Yau, "Eigenvalues and diameters for manifolds and graphs", pp. 79-105 in Tsing Hua lectures on geometry \& analysis (Hsinchu, 19901991), edited by S.-T. Yau, Int. Press, Cambridge, MA, 1997. MR 98h:58206 Zbl 0890.58093

[Colbois and El Soufi 2006] B. Colbois and A. El Soufi, "Eigenvalues of the Laplacian acting on p-forms and metric conformal deformations", Proc. Amer. Math. Soc. 134:3 (2006), 715-721. MR 2007e:58049 Zbl 1095.35013

[Colbois and Maerten 2008] B. Colbois and D. Maerten, "Eigenvalues estimate for the Neumann problem of a bounded domain”, J. Geom. Anal. 18:4 (2008), 1022-1032. MR 2009f:35243 Zbl 1158.58014

[Friedman and Tillich 2000] J. Friedman and J.-P. Tillich, "Laplacian eigenvalues and distances between subsets of a manifold", J. Differential Geom. 56:2 (2000), 285-299. MR 2002g:58050 Zbl 1032.58015

[Gentile and Pagliara 1995] G. Gentile and V. Pagliara, "Riemannian metrics with large first eigenvalue on forms of degree p", Proc. Amer. Math. Soc. 123:12 (1995), 3855-3858. MR 96b:58115 Zbl 0848.53022

[Grigor'yan et al. 2004] A. Grigor'yan, Y. Netrusov, and S.-T. Yau, "Eigenvalues of elliptic operators and geometric applications", pp. 147-217 in Surveys in differential geometry, vol. 9, edited by A. Grigor'yan and S. T. Yau, Int. Press, Somerville, MA, 2004. MR 2007f:58039 Zbl 1061.58027

[Gromov and Milman 1983] M. Gromov and V. D. Milman, "A topological application of the isoperimetric inequality”, Amer. J. Math. 105:4 (1983), 843-854. MR 84k:28012 Zbl 0522.53039

[Guerini 2004] P. Guerini, "Prescription du spectre du laplacien de Hodge-de Rham", Ann. Sci. École Norm. Sup. (4) 37:2 (2004), 270-303. MR 2005k:58063 Zbl 1068.58016

[Villani 2009] C. Villani, Optimal transport: Old and new, Grundlehren der Mathematischen Wissenschaften 338, Springer, Berlin, 2009. MR 2010f:49001 Zbl 1156.53003

Received December 14, 2009.

\author{
BRUNO COLBOIS \\ UNIVERSITÉ DE NEUCHÂTEL \\ INSTITUT DE MATHÉMATIQUES \\ RUE EMILE ARGAND 11 \\ CH-2007, NEUCHÂTEL \\ SWITZERLAND \\ bruno.colbois@unine.ch
}

\title{
ALESSANDRO SAVO
}

DiPARTIMENTO Di SCIENZE DI BASE E APPLICATE PER L'INGEGNERIA

SAPIENZA UNIVERSITÀ DI ROMA

VIA ANTONIO SCARPA 16

00161 RoMA

ITALY

savo@dmmm.uniroma1.it 


\title{
PACIFIC JOURNAL OF MATHEMATICS
}

\author{
http://www.pjmath.org \\ Founded in 1951 by
}

E. F. Beckenbach (1906-1982) and F. Wolf (1904-1989)

\section{EDITORS}

V. S. Varadarajan (Managing Editor)

Department of Mathematics

University of California

Los Angeles, CA 90095-1555

pacific@math.ucla.edu

Vyjayanthi Chari

Department of Mathematics

University of California

Riverside, CA 92521-0135

chari@math.ucr.edu

\section{Robert Finn}

Department of Mathematics Stanford University

Stanford, CA 94305-2125

finn@math.stanford.edu

Kefeng Liu

Department of Mathematics

University of California

Los Angeles, CA 90095-1555

liu@math.ucla.edu
Darren Long

Department of Mathematics

University of California

Santa Barbara, CA 93106-3080

long@math.ucsb.edu

Jiang-Hua Lu

Department of Mathematics

The University of Hong Kong

Pokfulam Rd., Hong Kong jhlu@maths.hku.hk

Alexander Merkurjev

Department of Mathematics University of California

Los Angeles, CA 90095-1555 merkurev@math.ucla.edu
Sorin Popa

Department of Mathematics

University of California

Los Angeles, CA 90095-1555

popa@math.ucla.edu

Jie Qing

Department of Mathematics

University of California

Santa Cruz, CA 95064

qing@ cats.ucsc.edu

Jonathan Rogawski

Department of Mathematics

University of California

Los Angeles, CA 90095-1555

jonr@math.ucla.edu

\section{PRODUCTION}

pacific@math.berkeley.edu

Silvio Levy, Scientific Editor Matthew Cargo, Senior Production Editor

ACADEMIA SINICA, TAIPEI

CALIFORNIA INST. OF TECHNOLOGY

INST. DE MATEMÁTICA PURA E APLICADA

KEIO UNIVERSITY

MATH. SCIENCES RESEARCH INSTITUTE

NEW MEXICO STATE UNIV.

OREGON STATE UNIV.

\section{SUPPORTING INSTITUTIONS}

STANFORD UNIVERSITY
UNIV. OF BRITISH COLUMBIA
UNIV. OF CALIFORNIA, BERKELEY
UNIV. OF CALIFORNIA, DAVIS
UNIV. OF CALIFORNIA, LOS ANGELES
UNIV. OF CALIFORNIA, RIVERSIDE
UNIV. OF CALIFORNIA, SAN DIEGO
UNIV. OF CALIF., SANTA BARBARA

UNIV. OF CALIF., SANTA CRUZ

UNIV. OF MONTANA

UNIV. OF OREGON

UNIV. OF SOUTHERN CALIFORNIA

UNIV. OF UTAH

UNIV. OF WASHINGTON

WASHINGTON STATE UNIVERSITY

These supporting institutions contribute to the cost of publication of this Journal, but they are not owners or publishers and have no responsibility for its contents or policies.

See inside back cover or www.pjmath.org for submission instructions.

The subscription price for 2011 is US \$420/year for the electronic version, and \$485/year for print and electronic.

Subscriptions, requests for back issues from the last three years and changes of subscribers address should be sent to Pacific Journal of Mathematics, P.O. Box 4163, Berkeley, CA 94704-0163, U.S.A. Prior back issues are obtainable from Periodicals Service Company, 11 Main Street, Germantown, NY 12526-5635. The Pacific Journal of Mathematics is indexed by Mathematical Reviews, Zentralblatt MATH, PASCAL CNRS Index, Referativnyi Zhurnal, Current Mathematical Publications and the Science Citation Index.

The Pacific Journal of Mathematics (ISSN 0030-8730) at the University of California, c/o Department of Mathematics, 969 Evans Hall, Berkeley, CA 94720-3840, is published monthly except July and August. Periodical rate postage paid at Berkeley, CA 94704, and additional mailing offices. POSTMASTER: send address changes to Pacific Journal of Mathematics, P.O. Box 4163, Berkeley, CA 94704-0163.

PJM peer review and production are managed by EditFLOW ${ }^{\mathrm{TM}}$ from Mathematical Sciences Publishers.

PUBLISHED BY PACIFIC JOURNAL OF MATHEMATICS

at the University of California, Berkeley 94720-3840

A NON-PROFIT CORPORATION

Typeset in IATEX

Copyright $(2011$ by Pacific Journal of Mathematics 


\section{PACIFIC JOURNAL OF MATHEMATICS}

Volume $249 \quad$ No. $2 \quad$ February 2011

A gluing construction for prescribed mean curvature

257

ADRIAN BUTSCHER

Large eigenvalues and concentration

271

BRUNO COLBOIS and ALESSANDRO SAVO

Sur les conditions d'existence des faisceaux semi-stables sur les courbes multiples primitives

JEAN-MARC DRÉZET

A quantitative estimate for quasiintegral points in orbits

LIANG-CHUNG HSIA and JosePh H. Silverman

Möbius isoparametric hypersurfaces with three distinct principal curvatures, II

ZEJUN HU and SHUJIE ZHAI

Discrete Morse theory and Hopf bundles

371

DMITRY N. KOZLOV

Regularity of canonical and deficiency modules for monomial ideals

ManOJ KUMmini and SATOSHI MURAI

$\mathrm{SL}_{2}(\mathbb{C})$-character variety of a hyperbolic link and regulator

WEIPING LI and QINGXUE WANG

Hypergeometric evaluation identities and supercongruences

LING LONG

Necessary and sufficient conditions for unit graphs to be Hamiltonian

H. R. Maimani, M. R. Pournaki and S. Yassemi

Instability of the geodesic flow for the energy functional

DOMENico PERrone

String structures and canonical 3-forms

CORBETT REDDEN

Dual pairs and contragredients of irreducible representations

BINYONG SUN

On the number of pairs of positive integers $x_{1}, x_{2} \leq H$ such that $x_{1} x_{2}$ is a $k$-th power

DOYCHIN I. TOLEV

Correction to the article A Floer homology for exact contact embeddings 